\title{
CONSUMO EXCESIVO DE ALCOHOL ¿EXISTEN DIFERENCIAS DE GÉNERO?
}

\author{
Marcel Elipe Miravet \\ Grupo Salusex. Dpto. de Psicología Básica, Clínica y Psicobiología \\ Universitat Jaume I, Castelló. \\ elipe@uji.es \\ Verónica Cervigón Carrasco \\ Grupo Salusex. Dpto. de Psicología Básica, Clínica y Psicobiología \\ Universitat Jaume I, Castelló. \\ cervigon@uji.es \\ Olga Fernández García \\ Grupo Salusex. Dpto. de Psicología Evolutiva y de la Educación \\ Universitat de València. \\ Olga.Fernandez-Garcia@uv.es \\ Verónica Estruch \\ Grupo Salusex. Dpto. de Psicología Evolutiva y de la Educación \\ Universitat de València. \\ veronicaestruch11@gmail.com \\ Rafael Ballester Arnal \\ Grupo Salusex. Dpto. de Psicología Básica, Clínica y Psicobiología \\ Universitat Jaume I, Castelló. \\ rballest@uji.es
}

Recepción Artículo: 20 septiembre 2021 Admisión Evaluación: 22 septiembre 2021 Informe Evaluador 1: 24 septiembre 2021 Informe Evaluador 2: 28 septiembre 2021 Aprobación Publicación: 01 octubre 2021

\section{RESUMEN}

El consumo de alcohol es una práctica muy extendida en nuestra sociedad, pese a sus nocivas consecuencias (intoxicaciones etílicas, accidentes de tráfico, reyertas, ITS, etc.). Aunque sus efectos afectan a toda la población, con independencia del género o la edad, las estrategias preventivas no siempre tienen en cuenta dicha diversidad; aspecto que, seguramente, reduce su eficacia. Dada la necesidad de contar con un perfil más concreto de quienes consumen alcohol de manera más riesgosa, el objetivo de este trabajo es conocer si existen diferencias, según el género y la edad, en la vivencia de algunas consecuencias derivadas del consumo de alcohol. Para ello, 60 participantes (35 mujeres y 25 hombres), con edades comprendidas entre los 22 y los 64 años, respondieron una encuesta sobre el consumo de sustancias. Los resultados muestran que el 100\% de hombres y el $97 \%$ de mujeres han consumido alcohol. Los primeros son los que más han vomitado por la ingesta de alcohol (52.8\%; $\left.{ }_{2}^{2}=4.91 ; p=.027\right)$, más veces han ido en estado de embriaguez al trabajo ( $\left.87.5 \% ;{ }^{2}=7.72 ; p=.005\right)$, y más veces han conducido borrachos (66.7\%; ${ }^{2}=7.88 ; p=.005$ ). Por otro lado, la única diferencia que existe en función de 


\section{CONSUMO EXCESIVO DE ALCOHOL ¿EXISTEN DIFERENCIAS DE GÉNERO?}

la edad se centra en la experiencia de haber vomitado, siendo mayor entre las personas que tienen más años. A la vista de los resultados, preocupa la cantidad de consecuencias de riesgo que están asociadas a un consumo excesivo de alcohol, especialmente para los hombres; destacando igualmente el indicador de vomitar según la edad. Es por eso que, a la hora de realizar intervenciones de prevención, debería tenerse en cuenta la perspectiva de género, ampliando de igual forma los esfuerzos a todas las edades, y no solamente centrar las campañas e intervenciones hacia los más jóvenes.

Palabras clave: alcohol; consecuencias de consumo; género; edad; perfil de riesgo.

\section{ABSTRACT}

Excessive alcohol consumption, are there gender differences? Alcohol consumption is a very widespread practice in our society, despite its harmful consequences (alcohol poisoning, traffic accidents, brawls, STIs, etc.). Although its effects affect the entire population, regardless of gender or age, preventive strategies do not always take such diversity into account; aspect that, surely, reduces its effectiveness. Given the need to have a more specific profile of those who consume alcohol in a riskier way, the goal of this work is to find out if there are differences, according to gender and age, in the experience of some consequences derived from alcohol consumption. For that purpose, 60 participants ( 35 women and 25 men), aged between 22 and 64, answered a survey on substance use. Results show that $100 \%$ of men and $97 \%$ of women have consumed alcohol. Men are who have vomited the most due to the alcohol intake (52.8\%; $\left.{ }^{2}=4.91 ; p=.027\right)$, the more times they have gone intoxicated to work (87.5\%; $\left.{ }^{2}=7.72 ; p=.005\right)$, and the more times they have driven drunk (66.7\%; $\left.2=7.88 ; p=.005\right)$. Additionally, the only difference that exists based on age is focused on the experience of having vomited, being greater among older people. According to the results, the amount of risk consequences associated with excessive alcohol consumption is concerning, especially for men; also highlighting the vomiting indicator according to age. That is why, when carrying out prevention interventions, gender perspective should be taken into account, expanding efforts to all ages in the same way, and not only focusing campaigns and interventions on the youngest.

Keywords: alcohol; consumption consequences; gender; age; risk profile

\section{INTRODUCCIÓN}

El alcohol, junto con el tabaco, es la sustancia psicoactiva más consumida de nuestro país (OEDA, 2021). El consumo de esta sustancia empieza en edades bastante tempranas. El estudio realizado por Husky et al. (2020) indica que, a la edad de 12 años, el 90\% de los encuestados ya habían probado el alcohol. Además, este mismo estudio revela que el $75 \%$ de los participantes consumen alcohol de forma habitual en sus vidas, entendiendo como consumo habitual un mínimo de una copa al mes. Estos datos ponen de manifiesto que el consumo de alcohol es una práctica continuada con los años, llevada a cabo tanto por jóvenes como por adultos (Husky et al., 2020; OEDA, 2021).

El consumo de alcohol suele estar ligado a celebraciones y reuniones sociales. Estudios como el de Aan Het Rot et al. (2008) y el de Harder et al. (2014) afirman que el consumo de alcohol está asociado tanto al aumento de estados de ánimo positivos como a un descenso de los estados de ánimo negativos. Ahora bien, existe cierta discrepancia con dichos estados de ánimo, pues diversos estudios encontraron que el hecho de beber alcohol junto con otras personas aumenta la percepción subjetiva de inclusión social y de pertenencia al grupo (Armeli et al., 2003). De forma similar, el estudio de Monk, Qureshi y Heim (2020) afirma que beber alcohol en grupo genera una percepción subjetiva de felicidad, más que un estado de ánimo positivo.

Además, se ha demostrado que el consumo de alcohol también está muy asociado con consecuencias negativas para la salud. Accidentes de tráfico, reyertas callejeras, agresiones sexuales, problemas de adicción ...; son solo alguno de los problemas que más se relacionan con un consumo continuado y/o excesivo de esta sustancia. Según el informe de 2017 de la DGT (Dirección General de Tráfico), en ese mismo año hubo un total de 2.115 conductores que se vieron implicados en accidentes mortales en las carreteras españolas. De ellos, 1.592 conductores (esto es, el 75\%) habían consumido más alcohol del permitido por la normativa vigente. Las peleas son 
otra de las consecuencias más frecuentes por el consumo abusivo de esta sustancia. El consumo excesivo de alcohol conlleva no solo a agresiones con personas desconocidas, sino que también puede llevar a disputas con personas conocidas, como son amigos, familiares o incluso parejas (Crane et al., 2019; Wells et al., 2005).

Además, diversos estudios ponen de manifiesto cómo el consumo de alcohol también está relacionado con un aumento de las agresiones sexuales hacia las mujeres (Fuentes-Pumarola et al., 2021; Hingsonet al., 2009). Por si fuera poco, el consumo de esta droga también incrementa las conductas sexuales de riesgo, como el hecho de no utilizar métodos de protección como el preservativo (Ruiz et al., 2020), lo que conlleva una mayor probabilidad de contraer infecciones de transmisión sexual (Rojas-Murcia et al., 2015), además de aumentar el número de embarazos no deseados (Rehm et al., 2012; Yaesoubi et al., 2021). Asimismo, varios estudios muestran como el consumo de bebidas alcohólicas se relaciona directamente con enfermedades como la hipertensión, la diabetes, 0 el hecho de padecer enfermedades cardíacas, como arritmias 0 ataques cardíacos (Suter y Tremblay, 2005; Haseeb et al., 2017).

Por otra parte, la literatura existente ha demostrado que el consumo de alcohol difiere entre mujeres y hombres. Estas diferencias las encontramos en distintos aspectos, como puede ser a nivel de prevalencias, factores de riesgo para iniciarse en el consumo, o las consecuencias que el consumo elevado puede tener. Así, el informe del Ministerio de Sanidad (OEDA, 2021), indica que la prevalencia del consumo de alcohol en hombres y mujeres se sitúa en el $95.5 \%$ y el $90.4 \%$, respectivamente. Además, fenómenos como el botellón ha sido practicado por el $46.5 \%$ de los hombres y el $37.2 \%$ de las mujeres en el año 2019. Aunque el citado informe del ministerio simplemente contempla datos de prevalencia, existen estudios como el de Dir et al. (2017), donde se analizan los motivos que llevan tanto a hombres como a mujeres a realizar consumos puntuales y excesivos de alcohol (binge drinking), encontrando como resultado la presión social a la que se enfrentan especialmente los hombres al estar en grupo, hecho que aumenta su consumo de alcohol. Por otro lado, estudios como el de Wilsnack et al. (2018) analizan las consecuencias a medio y largo plazo del consumo abusivo entre hombres y mujeres, encontrando que los hombres tienen más probabilidades de contraer enfermedades cardiacas, mientras que las mujeres padecen con más frecuencia enfermedades hepáticas. Este mismo estudio también analiza las consecuencias a nivel social y conductual, siendo ellos los que cometen más agresiones físicas y sexuales, mientras que las mujeres reciben mayor violencia en el hogar, tanto física como psicológica (Wilsnack et al., 2018).

Los resultados que reflejan estos estudios son realmente interesantes, dado que muestran los efectos a medio y largo plazo del consumo de alcohol, tanto para población general como para hombres y mujeres por separado. Sin embargo, apenas existen estudios que analizan cuales son las consecuencias de un consumo abusivo a corto plazo, y mucho menos analizando esas consecuencias en función del género de la población. Dada la escasez de datos y la importancia de estos efectos a corto plazo para el cambio de conducta y la prevención del consumo del alcohol, resulta necesario investigar estas consecuencias.

Por lo tanto, el principal objetivo de este trabajo es analizar las consecuencias que a corto plazo causa el consumo de alcohol en hombres y mujeres. De la misma forma, se quiere ver el posible papel que la edad tiene en el consumo de esta droga.

\section{MÉTODO}

\section{Participantes}

La muestra estaba formada por un total de 60 personas, 35 (54\%) de las cuales eran mujeres y 25 (46\%) hombres. La edad de los participantes estaba comprendida entre los 22 y los 64 años, con una media de 32 años (DT=9.9), siendo la media de los hombres de 34 años (DT=10.73) y la de las mujeres de 31.4 (DT=9.3). Del total de los participantes, el $13.6 \%$ eran estudiantes universitarios, el $79.7 \%$ eran trabajadores activos, el $5.1 \%$ estaban desempleados y el 1.7\% estaban jubilados. En cuanto a su situación personal, el 34\% de los encuestados eran solteros, el $42.3 \%$ tenían pareja estable, un 22\% estaban casados, y solamente el 1.7\% se había divorciado. 


\section{CONSUMO EXCESIVO DE ALCOHOL ¿EXISTEN DIFERENCIAS DE GÉNERO?}

\section{Instrumento}

Para la recogida de los datos se administró un cuestionario ad-hoc sobre consumo de sustancias, el cual estaba compuesto por un total de 50 ítems, los cuales estaban divididos en cuatro bloques temáticos (tabaco, alcohol, azúcar y cafeína). En este estudio en concreto se utilizó el bloque de consumo de alcohol. Para esta sustancia, había un total de 15 preguntas:

Tres ítems de formato abierto, los cuales preguntaban la edad de inicio de consumo de esta sustancia, cuando empezaron a consumirla de forma habitual, y la cantidad de bebida que consumen actualmente cuando beben.

Cuatro ítems dicotómicos que preguntan sobre ciertas conductas, como haber hecho "botellón"; y si han vomitado, conducido y trabajado/estudiado después de beber.

Cuatro ítems tipo Likert de 6 puntos que evaluaban la frecuencia actual de consumo.

Dos ítems tipo Likert de 6 puntos sobre hábitos de consumo (qué beben más a menudo y cuándo suelen hacerlo)

Dos ítems dicotómicos que evalúan si creen que actualmente consumen demasiado alcohol, y si han intentado reducir el consumo de esta sustancia.

\section{Procedimiento}

Los datos utilizados en esta investigación fueron recogidos en el mes de octubre del año 2020, una vez la investigación fue aprobada por la Comisión de Ética. La difusión de este estudio se llevó a cabo mediante redes sociales (Facebook, Instagram y WhatsApp). Una vez finalizada la difusión, la administración del cuestionario a Ios participantes fue mediante vía telemática, utilizándose para ello el correo institucional del investigador principal. En el correo de administración se proporcionó a los participantes el objetivo del estudio, la descripción de los objetivos y la duración aproximada que podría llevarles cumplimentar los formularios. Así mismo, se les informó que en todo momento se cumplían las medidas de protección de datos, solicitándoles de igual forma su consentimiento informado.

\section{Análisis estadísticos}

Los datos fueron almacenados y analizados en el programa estadístico SPSS (versión 26). Se realizaron análisis descriptivos para estudiar los hábitos de consumo de los participantes, así como los datos sociodemográficos.

También se realizaron análisis Chi cuadrado para ver si existían diferencias entre mujeres y hombres a la hora de realizar distintas conductas después de haber consumido alcohol, como son conducir un vehículo, ir en estado de embriaguez al trabajo o centro de estudio, vomitar tras consumir alcohol, etc. Finalmente se realizó una diferencia de medias (prueba $t$ de Student) para analizar si el hecho de vomitar por un consumo excesivo de alcohol cambia en función de la edad de los participantes.

\section{Resultados}

En cuanto al consumo de alcohol, tanto el 100\% los hombres como de las mujeres de este estudio indicaron que probaron por primera vez el alcohol a los 14 años y medio (DT=1.65), empezando a consumir alcohol de forma más habitual (al menos dos veces por semana) a los 18 años. En cuanto al consumo actual de alcohol según el género (ver Tabla 1), se observa cómo los hombres beben más a diario que las mujeres. Sin embargo, ellas afirman beber más los fines de semana. 
Tabla 1

Porcentajes de consumo de alcohol en muestra total y según género ( $n=60)$

\begin{tabular}{lccc}
\hline & Hombres (\%) & Mujeres (\%) & Total (\%) \\
\hline Cada día & 16 & 3 & 8.5 \\
Cada 2 días & 8 & 6 & 7 \\
Cada 3 días & 12 & 12 & 12 \\
Cada 4 días & 12 & 6 & 8.5 \\
Fines de semana o festivos & 52 & 73 & 64 \\
\hline
\end{tabular}

Por su parte, en la Tabla 2 se observa el tipo de bebida que consumen con mayor frecuencia los hombres y las mujeres. Mientras que los hombres se decantan más por la cerveza, las mujeres parecen escoger el vino como su bebida más habitual.

Tabla 2

Porcentajes de tipo de bebida alcohólica consumida en muestra total y según género $(n=60)$

\begin{tabular}{lccc}
\hline & Hombres (\%) & Mujeres (\%) & Total (\%) \\
\hline Cerveza & 84 & 67 & 74 \\
Vino & 12 & 27 & 21 \\
Cubatas & 0 & 3 & 2 \\
Cava & 4 & 0 & 2 \\
Licores & 0 & 3 & 1 \\
\hline
\end{tabular}

En cuanto a las características del consumo de alcohol, el $80 \%$ de los hombres y el $74 \%$ de las mujeres han practicado alguna vez el fenómeno conocido como "botellón", no existiendo diferencias entre ambos géneros en la realización de este fenómeno social ( $\left.{ }^{2}=.33 ; p=.564\right)$. En dicho acontecimiento, los hombres consumen una media de 6 cubatas ( $\mathrm{DT}=2.2$ ), mientras que las mujeres beben una media de $5(\mathrm{DT}=1.8)$, no existiendo diferencias significativas en la cantidad de combinados que consumen en ese acto ( $\mathrm{t}=1.82 ; p<.076)$.

Siguiendo con esta tónica de consumo, el 68\% de los hombres y el 53\% de las mujeres han tenido que irse a casa antes de tiempo debido a un consumo excesivo de alcohol. A pesar de la desigualdad que hay entre estos porcentajes, no existen diferencias significativas entre ellos.

Ahora bien, si hablamos de haber vomitado por un consumo excesivo de alcohol, el 100\% de los hombres de la muestra afirma haberlo hecho, siendo este porcentaje del 82.5\% para las mujeres, encontrándose en este caso diferencias significativas entre ambos géneros.

Por otra parte, el $56 \%$ de los hombres y el $20.6 \%$ de las mujeres afirman haber conducido un vehículo en estado de embriaguez, siendo estas diferencias entre géneros significativas. Además, son también los hombres quienes más veces han acudido al trabajo 0 al centro de estudios en estado de embriaguez (28\%), en comparación con las mujeres (3\%), siendo otra vez estas diferencias estadísticamente significativas. En la Figura 1 pueden observarse gráficamente estos resultados. 


\section{CONSUMO EXCESIVO DE ALCOHOL ¿EXISTEN DIFERENCIAS DE GÉNERO?}

Figura 1. Porcentajes de las consecuencias de un consumo abusivo de alcohol según el género.

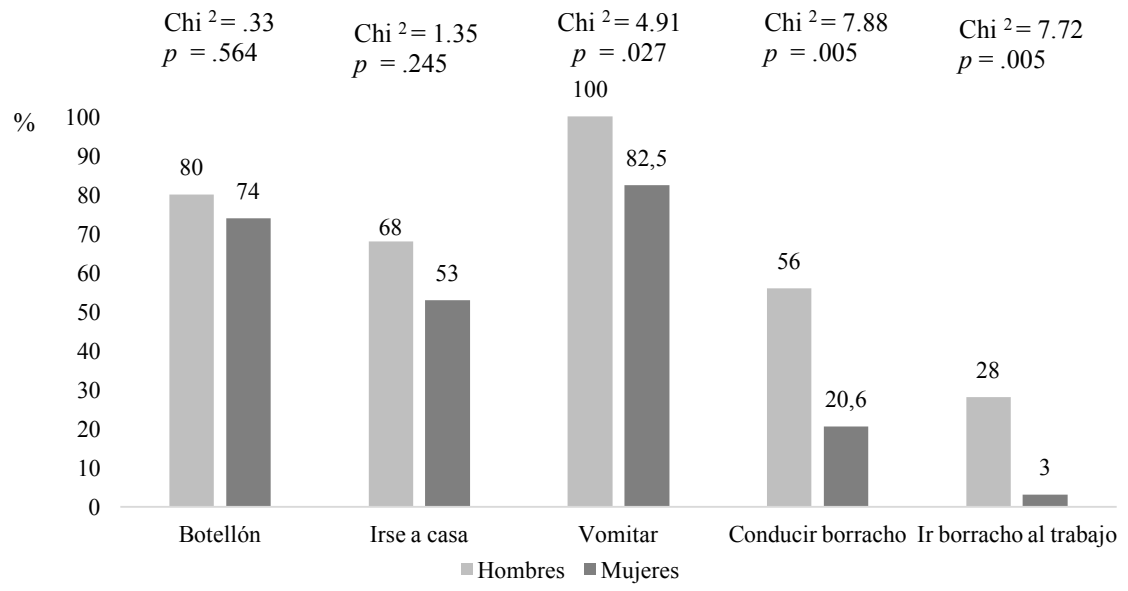

Por último, se quiso averiguar si la edad también era un componente a tener en cuenta en todas estas consecuencias de riesgo debidas a un consumo excesivo de alcohol, como las mencionadas anteriormente. La Tabla 3 muestra como solamente se encontraron diferencias significativas en el hecho de haber vomitado por una ingesta elevada de alcohol, siendo esta experiencia mayor tal y como aumenta la edad de los participantes.

Tabla 3

Media de edad y desviación según consecuencias de riesgo de consumo de alcohol ( $n=60)$

\begin{tabular}{lccc}
\hline & Sí $(M y D T)$ & No $(M y D T)$ & $\mathrm{t}$ \\
\hline Hacer "botellón" & $31(8.1)$ & $37(13.6)$ & 1.59 \\
Irse temprano a casa & $32.7(8.7)$ & $31.9(11.7)$ & .27 \\
Vomitar & $33.3(10)$ & $24.6(2.6)$ & $4.95^{* *}$ \\
Conducir un vehículo "borracho" & $32.7(9.6)$ & $32.2(10.2)$ & .861 \\
Acudir "borracho" al trabajo & $30(5.8)$ & $32.8(10.4)$ & .029 \\
\hline
\end{tabular}

Nota: $* *=p<.001$

\section{Discusión}

El principal objetivo de este estudio era analizar las principales características en el consumo de alcohol entre hombres y mujeres, así como las consecuencias que a corto plazo se asocian al consumo excesivo de esta sustancia.

Los resultados de prevalencia general de consumo de alcohol proporcionados por el Ministerio de Sanidad (el 93\%) van en la línea de los que aquí hemos encontrado (el 100\%), indicando que casi toda la población de nuestro país ha consumido en alguna ocasión bebidas alcohólicas (OEDA, 2021). En cuanto a la edad de inicio de consumo, Husky et al. (2020) encontraron que a los 12 años el 90\% de su muestra ya había consumido alcohol, por lo que los resultados que hemos encontrado (el 100\% a los 14 años) siguen la línea ascendente con los de dicho estudio.

En cuanto a la prevalencia por géneros, Ios principales datos los proporciona el Ministerio de Sanidad (OEDA, 2021). El 14\% de los hombres y el 3\% de las mujeres consumen alcohol diariamente, datos muy similares a los encontrados en este estudio (16\% y $3 \%$, respectivamente). Sin embargo, encontramos alguna dife- 
rencia en el tipo de bebida consumida con más frecuencia. En nuestro estudio, el 84\% de los hombres y el $67 \%$ de las mujeres afirma beber cerveza como bebida alcohólica más habitual, mientras que en la encuesta nacional estos porcentajes son del $49.5 \%$ en hombres y $28.1 \%$ en mujeres. Por su parte, el $12 \%$ de los hombres y el $27 \%$ de las mujeres afirman beber vino de forma habitual, mientras que los valores del ministerio son inversos a los nuestros, siendo del $24 \%$ en hombres y el $16 \%$ en mujeres. Finalmente, los combinados apenas se consumen de forma habitual por los participantes de este estudio ( $0 \%$ en hombres y $3 \%$ en mujeres), mientras que en la población española estos porcentajes ascienden al $18.3 \%$ y al $9.3 \%$, respectivamente.

En cuanto a las consecuencias generadas por el consumo de alcohol, la mayoría de las investigaciones se centran en aquellas que tienen efectos a medio o largo plazo. Sin embargo, los resultados del trabajo realizado por Grahan et al. (2011) están en línea con los que hemos encontrado nosotros, indicando que los hombres se enfrentan a más consecuencias en el trabajo por el hecho de consumir alcohol, lo cual tendría sentido si alguna vez han llegado en estado de embriaguez a su puesto laboral. Siguiendo esta misma línea, los estudios de Gassaye et al. (2015) y Gomes et al. (2019), encontraron que el 14.4\% y el 11.2\%, respectivamente, habían acudido al trabajo en estado de embriaguez; datos muy similares a los que aquí encontrados (13.6\% en nuestra muestra). Otros estudios, como el de Wilsnack et al. (2018) analizan otras conductas de riesgo, como conducir borrachos. Sus resultados van en línea con los que hemos hallado, dado que son los hombres quienes conducen con mayor frecuencia bajo los efectos del alcohol. En cuanto al acto de vomitar, el estudio de Mallett et al. (2011) concluye que el $45 \%$ de su muestra llegó a vomitar por el consumo excesivo de alcohol. Sin embargo, en nuestro estudio este porcentaje supera el $80 \%$ de los casos, tanto en hombres como en mujeres.

A pesar de la importancia de estos hallazgos, hay que remarcar algunas limitaciones de este trabajo. En primer lugar, se encontraría el bajo tamaño muestral. Aunque los resultados son innovadores, no se pueden generalizar los hallazgos aquí encontrados, pues se requieren nuevos estudios que corroboren estos resultados con un mayor número de participantes. Por otro lado, se preguntó por el consumo bruto de alcohol, no estandarizando la cantidad de alcohol consumido en unidades estándares, como pueden ser las Unidades de Bebida Estándar (UBE), o los gramos de alcohol puro ingeridos. Además, no se preguntó si además del alcohol se había consumido alguna otra droga que pudiera afectar al estado físico y/o mental de los sujetos.

No obstante, creemos que estos hallazgos destacan por su novedad en lo referente al consumo de alcohol. Sirviendo como complemento a las consecuencias a largo plazo que se han comentado en el marco teórico, los resultados que hemos encontrado reafirman el grave problema que supone el consumo elevado de alcohol a corto plazo, ya sea tanto para hombres como mujeres, como para personas jóvenes 0 adultas de nuestra sociedad. Atendiendo a estas diferencias de género y edad en el consumo, se plantea la necesidad de diseñar futuras estrategias de intervención, las cuales deberían tener en cuenta tanto la perspectiva de género como la edad. De esta forma, se mejorarían los resultados de estos programas, previniendo e informando tanto a hombres como a mujeres de las consecuencias que a corto plazo que tiene un consumo excesivo de alcohol. Aunque las intervenciones mayoritariamente se centran en los más jóvenes (en muchos casos con la finalidad de incrementar la gravedad percibida de un consumo excesivo en etapas tempranas), estas también deberían estar enfocadas hacia personas de mayor edad, puesto que los adultos también realizan consumos elevados, por lo que ampliar el rango de edad para aplicar estas intervenciones sería de gran utilidad.

\section{REFERENCIAS BIBLIOGRÁFICAS}

Aan het Rot, M., Russell, J., Moskowitz, D., \& Young, S. (2008). Alcohol in a Social Context: Findings From Event-Contingent Recording Studies of Everyday Social Interactions. Alcoholism: Clinical And Experimental Research, 32(3), 459-471. https://doi.org/10.1111/j.1530-0277.2007.00590.x

Armeli, S., Tennen, H., Todd, M., Carney, M., Mohr, C., Affleck, G., \& Hromi, A. (2003). A daily process examination of the stress-response dampening effects of alcohol consumption. Psychology Of Addictive Behaviors, 17(4), 266-276. https://doi.org/10.1037/0893-164x.17.4.266 


\section{CONSUMO EXCESIVO DE ALCOHOL ¿EXISTEN DIFERENCIAS DE GÉNERO?}

Crane, C., Schlauch, R., \& Miller, K. (2019). The association between caffeinated alcoholic beverages and the perpetration of intimate partner violence. The American Journal Of Drug And Alcohol Abuse, 45(5), 538-545. https://doi.org/10.1080/00952990.2019.1605522

Dirección General de Tráfico (DGT), Ministerio del Interior (2017). Siniestralidad relacionada con el consumo de alcohol y drogas: 2016-2017. Recuperado de: SINIESTRALIDAD RELACIONADA CON EL CONSUMO DE ALCOHOL Y DROGAS 2016-2017 (dgt.es)

Dir, A., Bell, R., Adams, Z., \& Hulvershorn, L. (2017). Gender differences in risk factors for adolescent binge drinking and implications for intervention and prevention. Frontiers In Psychiatry, 8. https://doi.org/10.3389/fpsyt.2017.00289

Fuentes-Pumarola, C., Reyes-Amargant, Z., Berenguer-Simon, A., Ballester-Ferrando, D., Burjalés-Martí, M., Rigol-Cuadra, A., Maestre-González, E., Rascón-Hernán, C., Galbany-Estragués, P., \& Rodríguez-Martín, D. (2021). Alcohol Use and Sexual Violence among Nursing Students in Catalonia, Spain: A Multicentre CrossSectional Study. International Journal Of Environmental Research And Public Health, 18(11), 6036. https://doi.org/10.3390/ijerph18116036

Gassaye, D., Bossali, F., Atipo-Ibara, B., Ahoui, C., Mouele, M., \& Ibara, J. (2015). Alcohol consummation prevailing in Brazzaville city in 2014. Journal Africain D’hépato-Gastroentérologie, 9(4), 160-162. https://doi.org/10.1007/s12157-015-0612-x

Gomes, K., Amato, T., Bedendo, A., Santos, E., \& Noto, A. (2019). Problems associated with binge drinking among students in Brazil's state capitals. Ciência \& Saúde Coletiva, 24(2), 497-507. https://doi.org/10.1590/1413-81232018242.35452016

Graham, K., Bernards, S., Knibbe, R., Kairouz, S., Kuntsche, S., \& Wilsnack, S., Greenfield, P., Obot, I., \& Gmel, G. (2011). Alcohol-related negative consequences among drinkers around the world. Addiction, 106(8), 1391-1405. https://doi.org/10.1111/j.1360-0443.2011.03425.x

Harder, V., Ayer, L., Rose, G., Naylor, M., \& Helzer, J. (2014). Alcohol, Moods and Male-Female Differences: Daily Interactive Voice Response over 6 Months. Alcohol And Alcoholism, 49(1), 60-65. https://doi.org/10.1093/alcalc/agt069

Haseeb, S., Alexander, B., \& Baranchuk, A. (2017). Wine and Cardiovascular Health. Circulation, 136(15), 14341448. https://doi.org/10.1161/circulationaha.117.030387

Hingson, R., Zha, W., \& Weitzman, E. (2009). Magnitude of and Trends in Alcohol-Related Mortality and Morbidity Among U.S. College Students Ages 18-24, 1998-2005. Journal Of Studies On Alcohol And Drugs, Supplement, (s16), 12-20. https://doi.org/10.15288/jsads.2009.s16.12

Mallett, K., Varvil-Weld, L., Turrisi, R., \& Read, A. (2011). An examination of college students' willingness to experience consequences as a unique predictor of alcohol problems. Psychology Of Addictive Behaviors, 25(1), 41-47. https://doi.org/10.1037/a0021494

Monk, R., Qureshi, A., \& Heim, D. (2020). An examination of the extent to which mood and context are associated with real-time alcohol consumption. Drug And Alcohol Dependence, 208, 107880. https://doi.org/10.1016/j.drugalcdep.2020.107880

Nolen-Hoeksema, S., \& Hilt, L. (2006). Possible Contributors to the Gender Differences in Alcohol Use and Problems. The Journal Of General Psychology, 133(4), 357-374. https://doi.org/10.3200/genp.133.4.357374

Observatorio Español de las Drogas y las Adicciones, Ministerio de Sanidad (2021). Alcohol, tabaco y drogas ilegales en España. Recuperado de: INFORME 2021. Alcohol, tabaco y drogas ilegales en España (sanidad.gob.es)

Rojas-Murcia, C., Pastor, Y., \& Esteban-Hernández, J. (2015). Ilusión de invulnerabilidad, estereotipos y per cepción de control del SIDA en universitarios [IIlusion of invulnerability, stereotypes and perception of con trol to AIDS in undergraduate students]. Revista Iberoamericana de Psicología y Salud, 6(1), 28-38. http://doi.org/10.1016/S2171-2069(15)70004-9 
Ruiz-Palomino, E., Gil-Llario, M., Giménez-García, C., \& Ballester-Arnal, R. (2020). Explanatory Psychological Factors of Inconsistently Condom Use among Spanish University Students: Gender Differences. The Spanish Journal Of Psychology, 23. https://doi.org/10.1017/sjp.2020.14

Suter, P., \& Tremblay, A. (2005). Is alcohol consumption a risk factor for weight gain and obesity?. Critical Reviews In Clinical Laboratory Sciences, 42(3), 197-227. https://doi.org/10.1080/10408360590913542

Rehm, J., Shield, K., Joharchi, N., \& Shuper, P. (2012). Alcohol consumption and the intention to engage in unprotected sex: systematic review and meta-analysis of experimental studies. Addiction, 107(1), 51-59. https://doi.org/10.1111/j.1360-0443.2011.03621.x

Wells, S., Graham, K., Speechley, M., \& J. Koval, J. (2005). Drinking patterns, drinking contexts and alcoholrelated aggression among late adolescent and young adult drinkers. Addiction, 100(7), 933-944. https://doi.org/10.1111/j.1360-0443.2005.001121.x

Wilsnack, R. W., Wilsnack, S. C., Gmel, G., \& Kantor, L. W. (2018). Gender differences in binge drinking: Prevalence, predictors, and consequences. Alcohol Research: Current Reviews, 39(1), 57-76.

Wolaver, A. (2002). Effects of heavy drinking in college on study effort, grade point average, and major choice. Contemporary Economic Policy, 20(4), 415-428. https://doi.org/10.1093/cep/20.4.415

Yaesoubi, R., Mahin, M., Martin, G., Paltiel, A., \& Sharifi, M. (2021). Reducing the Prevalence of Alcohol-Exposed Pregnancies in the United States: A Simulation Modeling Study. Medical Decision Making, 0(0), 1-11. https://doi.org/10.1177/0272989x211023203 
